\title{
Modeling answer seize rate (ASR) based on redial calls attempt
}

\section{${ }^{1}$ Mohammadali Pourmina, ${ }^{2}$ Saeid Khandani and ${ }^{3}$ Farbod Razazi}

\author{
${ }^{1}$ Department of Communication Engineering, Science and Research Branch, Islamic Azad \\ University, Tehran, Iran, e-mail: pourmina@srbiau.ac.ir \\ ${ }^{2}$ Student of Science and Research Branch, Islamic Azad University, Tehran, Iran, \\ e-mail: saeidkhan120@yahoo.com \\ ${ }^{3}$ Department of Communication Engineering, Science and Research Branch, Islamic Azad \\ University, Tehran, Iran, e-mail: farbod.razzazi@gmail.com
}

\begin{abstract}
-
This Paper studies the effect of redial calls attempt on answer seize rate parameter and we show new modeling of ASR. In previous analysis of ASR only the expected value of successful calls was expressed as ASR value. ASR is the most important parameter in the teletraffic measurement. Because it has one by one relation between charging and subscriber's satisfaction. The ASR parameter expresses the successfully rate in all of the our trying at the communication set (subscriber-switch-transfer to access the desired result. Then studying about it is very important.
\end{abstract}

Keywords: Radial calls, teletraffic

\section{INTRODUCTION}

The sketch of ASR based on redial calls attempt will reach us to capable result that guide us to improve ASR. A very interesting note about ASR that we emphasize on it, is that: when number of redial calls attempt increase, ASR rate decreases and the best value of ASR is in the first and second calls attempt. Then the first and second calls attempt is more important for us .we describe this : that how successfully calls play positive feedback role to improve ASR and reciprocal unsuccessfully calls play to decrease ASR. The importance of ASR especially distinguished at traffic pick hours.

When our resource is finite and we most support all of subscriber demand .Here, ASR parameter effects on availability parameter.

For simulating this parameter at first we consider subscribers behavior. The probability distribution function and statistical model of subscriber behavior can help us to simulate this parameter as good as possible .when we observe the number of calls $(\mathrm{N})$ with number of calls Completed (M) ASR is shown Completed (M) ASR is shown:

$$
\mathrm{N} \leq \% \quad \text { where } \quad \mathrm{M} \frac{M}{N} \quad \text { ASR }=
$$

Apparently one unsuccessfully call in our set ( $N$ records) decrease ASR rate about $\frac{1}{N}$. But in fact we observe Another behavior. we suppose $\mathrm{K}$ unsuccessfully records belonged to one subscriber. Now if this subscriber decided to answer at second calls (then K-2 unsuccessfully call records will be removed from our set and then :

$$
\% \quad \text { where } \frac{M+1}{N-(k-2)} \text { ASR }=2<
$$

Now if we increase time and register $\mathrm{N}$ records then :

$$
\% \frac{(M+1)+[((M+1) / N))(K-2)]}{N} \quad \text { ASR }=
$$

We show how successfully calls play positive feedback role to improve ASR and reciprocal unsuccessfully calls play to decrease ASR.

Unfortunately Unsuccessful calls in addition to decrease ASR increasing seize, for example: especially when one route is blocked or cutting off the seize number of this route decrease about 2, 3or four times. And it is very important at traffic pick hours. Because our source can not handle this number of seize.

If at one route ASR is $\% 50$ and number of seize was 2000 record. Then number of answer is 1000 records. Now if we attempt to improve ASR about $\% 66$ then all of seize become 1515 records and then 485 unsuccessfully records removed from our set. Because only the result or answer was desired for us.

A Very interesting question which we love to answer is: when one subscriber attempt call is not completed. What is the probability that caller decides to redial and how many does he or she attempts call? Answer 
to this question expresses in the subscriber set behaviors. Where the subscriber attempt calls, until

\begin{tabular}{|c|c|c|c|}
\hline \multicolumn{4}{c}{ Table of fits } \\
\hline Type & R-square & RMSE & Adj R-sq \\
\hline \begin{tabular}{c|c|c|c|}
\hline Expon & 0.9937453905 & 32.016191 & 0.9924944 \\
ential & 241677 & 14204013 & 68629 \\
\hline
\end{tabular}
\end{tabular}

the probability gets answer near the ASR rate.

\section{SUBSCRIBER BEHAVIOR}

When subscriber calls attempt is not completed at first time the caller maybe attempts new call after a short time. After several redial, if the call is not completed the caller decides to drop the call attempt. When we observe the calls attempt we will see that the number of redial calls attempt decrease exponentially .on the other hand subscriber behavior show when first call is not completed the subscriber that decide to attempt new call decrease and when second attempt is not completed the subscriber who decides to attempt new call decreasing and decreasing.....

Then the subscriber that attempt only one time, has the maximum number of calls attempt in our observation .Fortunately, this subscriber has the maximum value of ASR .then if the number of redial calls attempt increase the number of calls attempt and ASR value decrease.

In simulation of ASR sketch, we can show various statistical models such as exponentially,...

The probability density function (PDF) of an exponentially distribution has the form

$$
\mathrm{x}) \lambda \operatorname{EXP}(-\lambda)=\lambda \mathrm{F}(\mathrm{x} \text {, }
$$

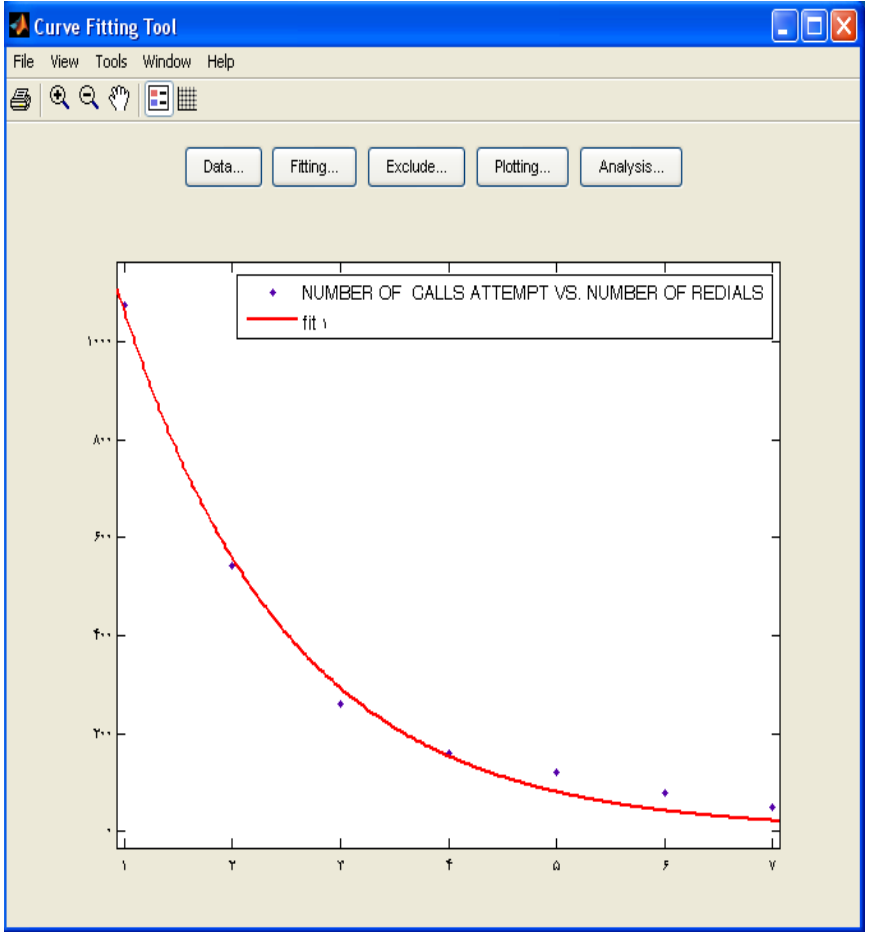

$$
f(x)=a^{\star} \exp \left(b^{\star} x\right)
$$

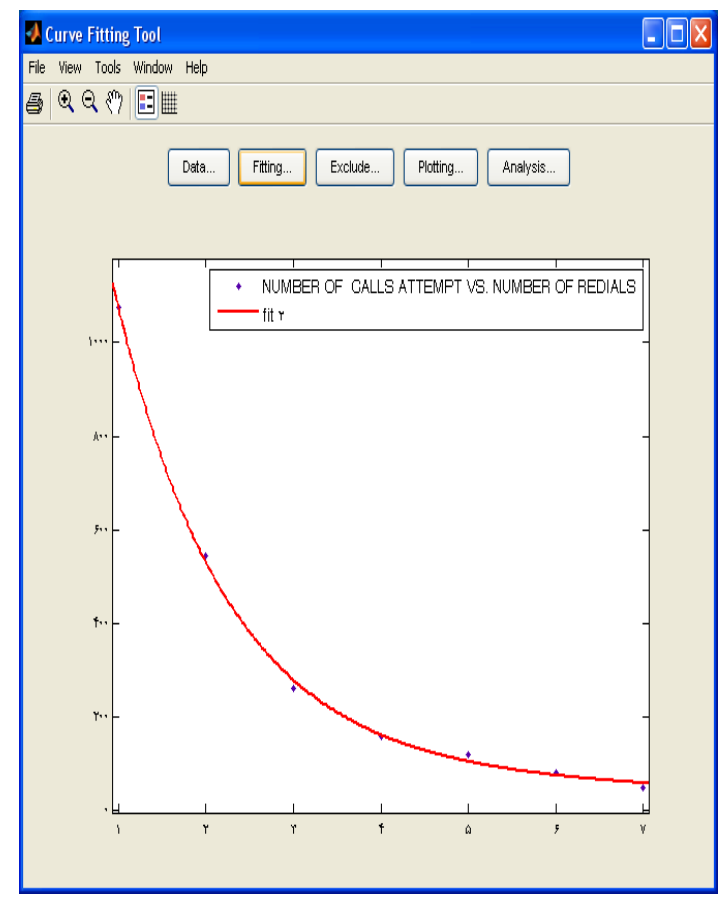

$f(x)=a^{*} \exp \left(b^{\star} x\right)+c^{*} \exp \left(d^{*} x\right)$ 
Am. J. Sci. Ind. Res., 2011, 2(2): 144-148

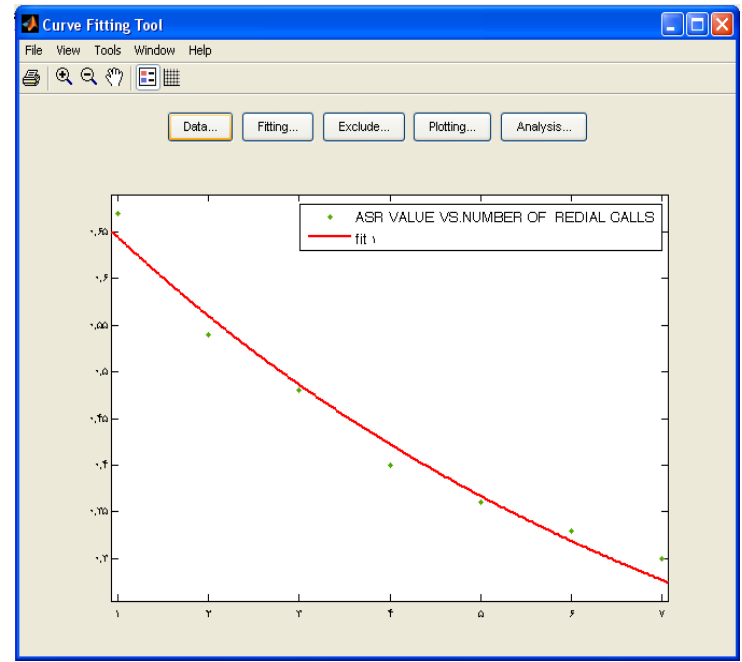

$f(x)=a^{*} \exp \left(b^{\star} x\right)$

\section{Table of fits}

\begin{tabular}{|c|c|c|c|}
\hline Type & R-square & RMSE & Adj R-sq \\
\hline $\begin{array}{c}\text { Expone } \\
\text { ntial }\end{array}$ & 0.9779610 & 0.0214310680 & 0,97355331 \\
\hline
\end{tabular}

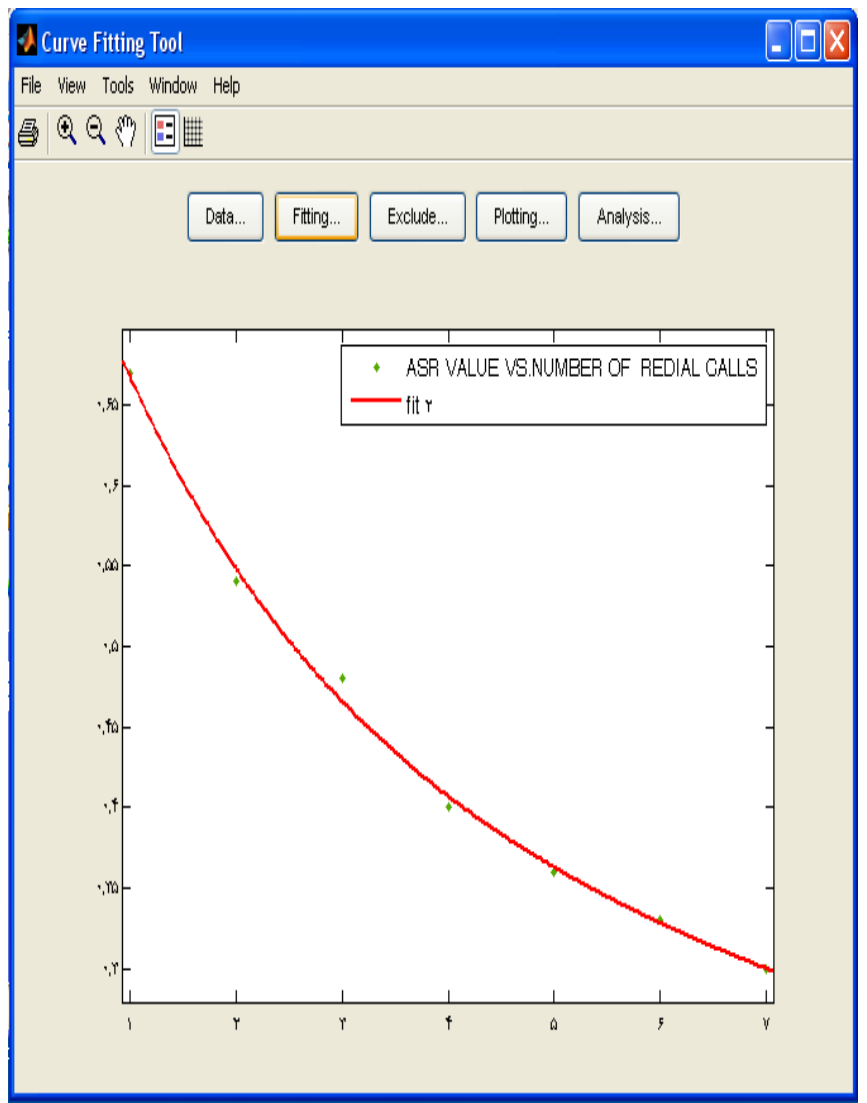

Table of fits

\begin{tabular}{|l|c:c|c|}
\hline Type & R-square & RMSE & Adj R- \\
& & & Sq \\
\hline Expon & 0.998948 & 16.947853 & 0.99789 \\
ential & 42191 & 6098 & 68 \\
\hline
\end{tabular}

$$
f(x)=a^{\star} \exp \left(b^{\star} x\right)+c^{\star} \exp \left(d^{\star} x\right)
$$

\section{PROBABILITY DISTRIBUTION CURV}

Most of the common probability distribution curves are explained by two parameters, that may be mean and variance the mean is expected value and it was middle point on the probability curve and variance used to describe dispersion of the probability curves. And it tells us how the values are dispersed about the center or mean of the curve.

$$
s=\sqrt{\frac{1}{n-1} \sum_{i=1}^{n}\left(x_{i}-\bar{x}\right)^{2}}
$$

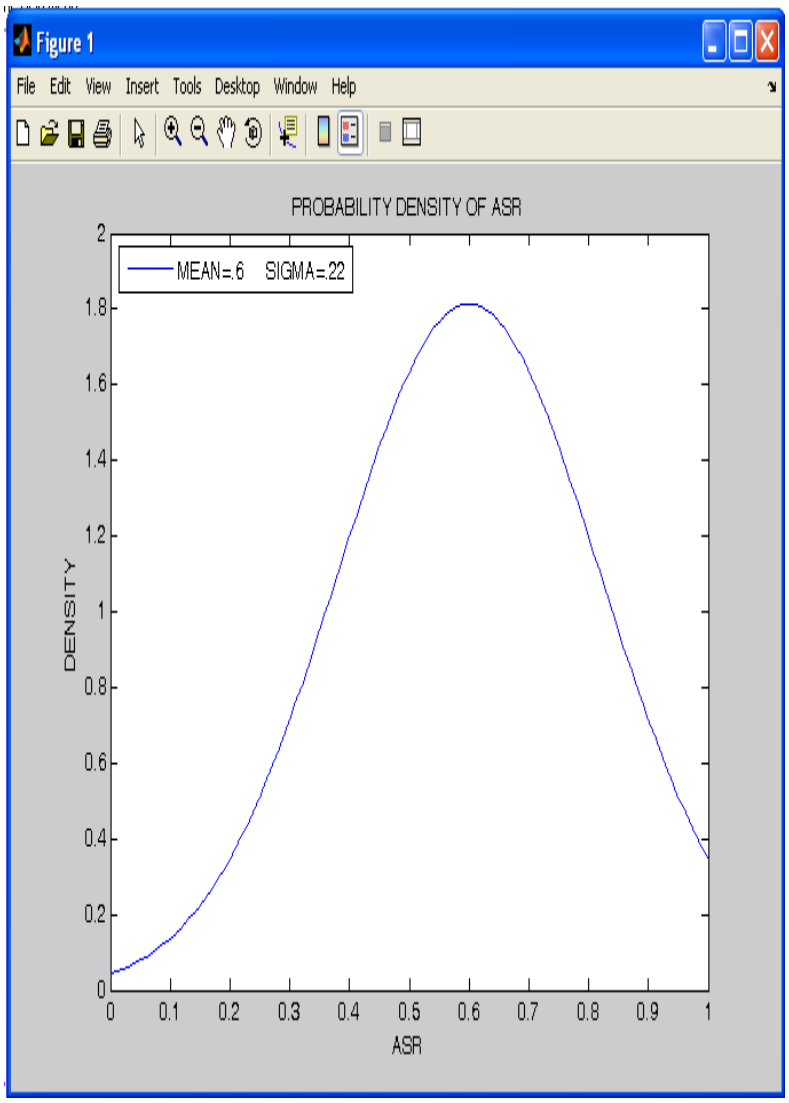




\begin{tabular}{|c|ccc|}
\hline \multicolumn{4}{|c}{ Table of fits } \\
\hline Type & R-square & RMSE & Adj R-sq \\
\hline Expone & 0.9968366 & 0.0104821211 & 0,99367323 \\
ntial & 16225 & 1688665 & 24505 \\
\hline
\end{tabular}

\section{STATISTICAL ANALYSIS OF CALL COMPLETION PROBABILITY}

$\mathrm{n}$ this section we study call completion Probability and we show that call's attempt are not Independent from another at distinct period of time. Because if call's attempt were independent from another, ASR rate must be constant at one period of time. But in fact we observe that call's probability and ASR rate decrease when call's attempt increase. On the other hand subscriber's calls attempt influences from another subscribers calls attempt ,specially influence from his or her calls attempt. Because it is possible that several subscribers decide to call with one subscriber at destination in one period of time. event that we met usually happen and influence ASR.

The main reason for low ASR rate with increasing redial calls attempt for high traffic subscribers is that when one subscriber call becomes successful, another calls attempt is blocked due to subscriber busy, and until this call ends, every attempt decreases ASR .Here increasing access holding time $(A H T)$ can decrease ASR. In fact when AHT decreases for this subscriber ASR increases exponentially.

We continue our studies with one numerical example. We consider subscriber that have two calls attempt and We suppose:

Probability of call completed at first
attempt $=$$\frac{2}{3}$

Then :

Probability of call is not completed at first attempt $=\frac{1}{3}$

Probability of call completed at second attempt=P And

Probability of call is not completed at second attempt= $1-p=q$

Then all states are: no completed calls $[(1 / 3) q]$. Only one completed call $[(2 / 3) \mathrm{q}$ or $(1 / 3) \mathrm{p}]$ and two completed call $[(2 / 3) \mathrm{p}]$ Then:

ASR $=($ completed calls) $/$ (all calls) or $p+2 \frac{1}{3} q+\frac{2}{3} \quad$ ASR $=\left(\left(p \frac{2}{3}\right)\right) / 2(1)$

If probability of completed call at second attempt was as first $(p=2 / 3)$. then ASR rate was constant. But in fact ASR is not constant. Now if we consider ASR equal $1 / 2$ for second attempt then it consequently from (1): $\quad p=1 / 3$ and then $q=2 / 3$ for second attempt When we have 3 calls attempt then we have:

$\mathrm{ASR}=\left[3\left(\frac{2}{9} p\right)+2\left(\frac{2}{9} q\right)+2\left(\frac{4}{9} p\right)+\left(\frac{4}{9} q\right)+2\left(\frac{1}{9} p\right)+\left(\frac{1}{9} q\right)+\left(\frac{2}{9} p\right)\right] / 3$

The first phrase in second eq. express probability that all tree calls attempt were successful then its coefficient is 3 .

the probability of call was success at first attempt was $\frac{2}{3}$

And the probability of successful at second attempts was $\frac{1}{3}$ then if we suppose

Probability of call success at third attempts is $p$ then for tree calls successful we have:

$$
\begin{gathered}
\left.\mathrm{p3}=()^{\star \mathrm{p}} \quad \frac{1}{3}\right)^{\star}\left(\frac{2}{3}\right. \\
\quad \frac{P+1}{3} \text { Then } \quad \text { ASR }=
\end{gathered}
$$

Consequently for $\mathrm{n}$ redial attempts we have:

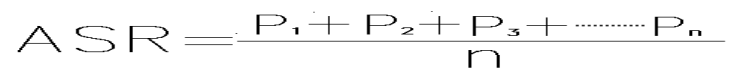

The probability of call completion at first attempt for high traffic subscribers usually is lower than low traffic subscriber's .

\section{CONCLUSION}

ASR is the most important parameter in teletraffic measurement .In this paper we consider this parameter variable with redial calls attempt. The variability of this parameter can help us to understand a better sense about traffic observation, especially we can consider this information to sketch better and better plan at future.

By ASR sketch we can predict calls successfully rate based on redial calls attempt.

Also ASR exponentially sketch tell us and our subscribers that their chance to complete calls Will decrease when redial calls attempt increase. then we can get the better and better desired result in future paper we show the best period time that has maximum chance of successful call rate .we saw 
Am. J. Sci. Ind. Res., 2011, 2(2): 144-148

when AHT decrease for high traffic subscriber, ASR value increase, consequently we can improve national and international ASR rate with decrease local and tolls AHT.

\section{REFERENCES}

[1]. Christopher D. Carothers, Richard M. Fujimto and YiBinglin "A Re-dial model for personal communications services networks" , IEEE 1995.

[2].Athanasios Papoulis and S. Unnikrishna Pillai ," Probability, random variables and stochastic processes", 2002. 